\title{
Large eddy simulation for jet noise: azimuthal decomposition and intermittency of the radiated sound
}

\author{
Guillaume A. Brès*, \\ Cascade Technologies Inc., Palo Alto, CA 94303, USA \\ Vincent Jaunet† Maxime Le Rallic; Peter Jordan§ \\ Institut PPRIME, CNRS-Université de Poitiers-ENSMA, Poitiers, France \\ Aaron Towne $\ddagger$ Oliver T. Schmidt † Tim Colonius \\ California Institute of Technology, Pasadena, CA 91125, USA \\ André A. V. Cavalierill \\ Instituto Tecnológico de Aeronáutica, São José dos Campos, Brazil \\ and \\ Sanjiva K. Lele** \\ Stanford University, Stanford, CA 94305
}

\begin{abstract}
To improve understanding and modeling of jet-noise source mechanisms, extensive experimental and numerical databases are generated for an isothermal Mach 0.9 turbulent jet at Reynolds number $R e=10^{6}$. The large eddy simulations (LES) feature localized adaptive mesh refinement, synthetic turbulence and wall modeling inside the nozzle to match the fully turbulent nozzle-exit boundary layers in the experiments. Long LES databases are collected for two grids with different mesh resolutions in the jet plume. Comparisons with the experimental measurements show good agreement for the flow and sound predictions, with the far-field noise spectra matching microphone data to within $0.5 \mathrm{~dB}$ for most relevant angles and frequencies. Preliminary results on the radiated noise azimuthal decomposition and temporal intermittency are also discussed. The azimuthal analysis shows that the axisymmetric mode is dominant at the peak radiation angles and that the first 3 Fourier azimuthal modes of the LES data recover more than $97 \%$ of the total acoustic energy at these angles. The temporal analysis highlights the presence of recurring intermittency in the radiated sound for the low-frequency range and main downstream angles. At these frequencies and angles, temporally-localized bursts of noise can reach levels up to 3 or $4 \mathrm{~dB}$ higher (or lower) than the long-time average.
\end{abstract}

\section{Nomenclature}

\begin{tabular}{|c|c|c|c|}
\hline$c$ & Speed of sound & $M$ & Mach number \\
\hline$D$ & Nozzle exit diameter & $M_{\infty}$ & Wind-tunnel Mach number \\
\hline$d t$ & Time step & $m$ & Azimuthal mode number \\
\hline$f$ & Frequency & $N P R$ & Nozzle pressure ratio \\
\hline$* *$ & $\begin{array}{l}\text { Research Scientist } \\
\text { toral Scholar } \\
\text { te Student } \\
\text { h Scientist } \\
\text { or, Dept. of Mechanical En } \\
\text { tt Professor, Divisão de En } \\
\text { or, Dept. of Mechanical En }\end{array}$ & te Fellow & \\
\hline
\end{tabular}

$$
1 \text { of } 16
$$

American Institute of Aeronautics and Astronautics 


$\begin{array}{ll}N T R & \text { Nozzle temperature ratio } \\ n_{x}, n_{r}, n_{\theta} & \text { Dimensions of the structured grids } \\ p & \text { Pressure } \\ R e & \text { Reynolds number } \rho U_{j} D / \mu \\ S t & \text { Strouhal number } f D / U_{j} \\ T & \text { Temperature } \\ t & \text { Time } \\ t_{s i m} & \text { Total simulation time } \\ U_{j} & \text { Mean streamwise jet velocity } \\ u, v, w & \text { Fluid velocity components } \\ \mathbf{x} & \text { Far-field microphone position } \\ x, y, z & \text { Cartesian coordinates } \\ \Delta t & \text { Sampling period } \\ \mu & \text { Dynamic viscosity }\end{array}$

$\begin{array}{ll}\phi & \text { Jet inlet angle } \\ \rho & \text { Density } \\ \theta & \text { Azimuthal angle } \\ \text { Subscript } & \\ \infty & \text { Free-stream property } \\ t & \text { Total (stagnation) property } \\ j & \text { Fully-expanded jet conditions } \\ \text { Superscript } \\ \sim & \text { Temporal Fourier-transformed quantity } \\ * & \text { Dimensional quantity } \\ , & \text { Disturbance quantity } \\ \dagger & \text { Complex conjugate } \\ - & \text { Time average }\end{array}$

\section{Introduction}

As reviewed by Jordan \& Colonius, ${ }^{1}$ there have been great advances in the past few years in identifying the physical mechanisms underlying the peak noise radiation to aft angle - namely the existence of convective, large-scale, turbulent wavepacket structures that directly radiate to the far-field. While the observation of wavepackets and the corresponding theory date to the earliest days of jet noise research, ${ }^{2,3}$ the recent breakthroughs were made possible in large part by progress in both experimental diagnostics (principally microphone arrays) and high-performance computations (now providing high-resolution, time-resolved flow data).

In this context, the present work is a continuation of a collaborative effort to improve understanding and modeling of the turbulent sources of sound in high-speed jets, through the use of such advanced experimental and numerical databases. The configuration investigated corresponds to an isothermal Mach 0.9 turbulent jet issued from a convergent-straight nozzle, with diameter-based Reynolds number $R e=10^{6}$. The experiments conducted at PPRIME Institute include characterization of the nozzle-exit boundary layers, flow field PIV, and both near-field and far-field pressure measurements on large microphone arrays. Companion large eddy simulations (LES) are performed at the same operating conditions, using the compressible flow solver "Charles" developed at Cascade Technologies.

In previous work, ${ }^{4,5}$ a systematic parametric study of the separate and combined effects of different modeling within the nozzle interior was conducted. The study focused on localized near-wall adaptive mesh refinement inside the nozzle, and synthetic turbulence and wall modeling applied on the nozzle internal surfaces, ${ }^{6,7}$ to replicate the effect of boundary layer trip present in the experiment and ensure fully turbulent profiles at the nozzle exit. Overall, the results showed significant improvement for both flow field and noise predictions when modeling inside the nozzle was applied, compared to the typical approach based on coarse resolution in nozzle and laminar flow assumption commonly used in most jet simulations. Based on that work, the simulations with modeling are extended for two grids with different mesh resolutions in the jet plume, to generate long LES databases for further analysis and data mining. Comprehensive details of the LES databases and comparisons with the available experimental measurements are presented in Sections II and III, respectively. Postprocessing and analysis of the databases are discussed in Section IV, including preliminary results on the noise azimuthal decomposition and intermittency.

\section{Description of the experimental and numerical databases}

\section{A. Flow configuration and experimental setup}

The study focuses on an isothermal Mach 0.9 jet issued from contoured convergent-straight nozzle of exit diameter $D=50 \mathrm{~mm}$. The experiments were performed in the anechoïc jet-noise facility of the PPRIME Institute at the Centre d'Études Aérodynamiques et Thermiques (CEAT), Poitiers, France. Transition is forced using an azimuthally homogeneous carborundum strip located 2.8 nozzle diameters upstream of the nozzle exit plane. The experimental diagnostics feature particle image velocimetry (PIV) for extensive measurement of the jet plume (see figure 1(a)), a 48-microphone near-field cage array for the eduction of 


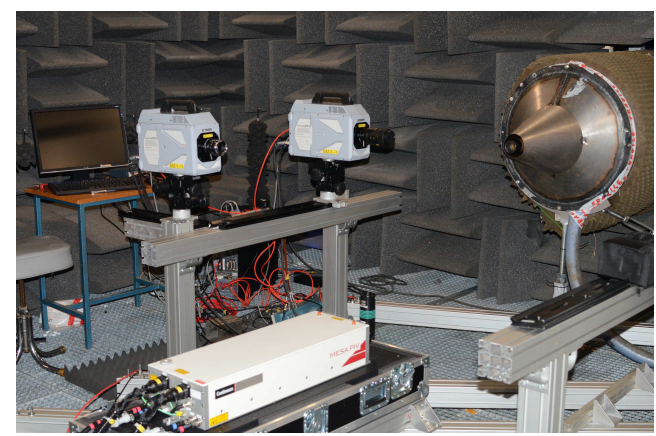

(a) PIV system

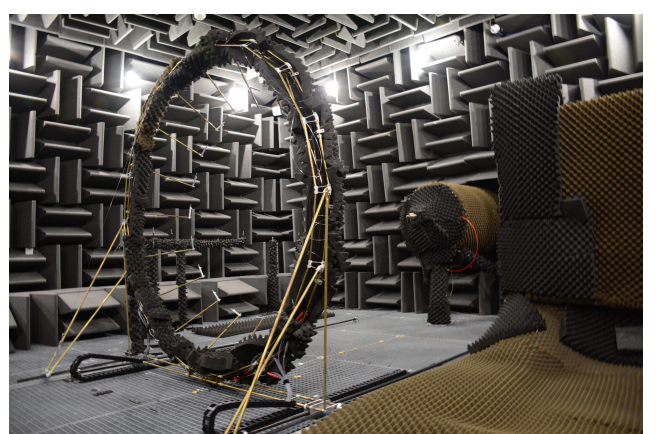

(b) 18-microphone azimuthal array

Figure 1. Experimental setup in the anechoïc jet-noise facility at PPRIME Institute

wavepacket signatures (upcoming measurements), and an axially traversable, 18-microphone, azimuthal array providing measurements of the sound field on a cylindrical surface of radius $r / D=14.3$ (see figure $1(b)$ ). Pressure measurements are also performed in the far field at a constant distance of $50 \mathrm{D}$ from the nozzle exit using a single microphone every $10^{\circ}$, from jet inlet angle $\phi=90^{\circ}$ (i.e., normal to nozzle-exit plane) to $160^{\circ}$ (i.e., downstream of nozzle exit).

The PIV system consisted of a Photron SAZ camera and a 532nm Continuum MESA PIV laser providing $6 \mathrm{~mJ}$ of light pulse energy. The system was placed on a traverse parallel to the jet axis in order to scan the jet flow field from axis location close to the nozzle up to 20 jet diameter. The camera was equipped with a $100 \mathrm{~mm}$ Macro lens with low optical distortion, with an aperture set at $\mathrm{f} \# 4$ to ensure sharpness of the particule images. Both the jet flow and the surrounding air were seeded using glycerin smoke particles, whose diameter lay in the range $1-2 \mu \mathrm{m}$ : thus sufficiently small to follow the velocity fluctuations of interest in this paper. The optical set-up gives diffraction limited particle size of $6 \mu \mathrm{m}$, smaller than a pixel size, but the combination of the particle size together with high energy laser formed particle images of 2-3 pixels in diameter. Hence, no evidence of peak-locking was found in the data set.

Two different fields of view (FOV) were used during the experimental campaign. The first FOV measured the velocity field in an area of about $2 D \times 2 D$, and was used for axial positions from the nozzle exit up to $x=6 D$. The second FOV measured the velocity field in an area of $4 D \times 4 D$, and was used for more downstream locations, i.e., $x>5 D$. Hence, a finer spatial resolution was obtained for measurement close to the nozzle exit to ensure good resolution of the local velocity gradients. The complete measurement of the jet flow was obtained with the use of 11 acquisitions performed at various downstream locations. In between each of these locations an overlap of $20 \%$ of the FOV was set in order to control the correct alignment of the measured velocity fields. A calibration was made at all acquisition positions in order to be able to correct for both the remaining optical distortions and laser light sheet/measurement plane misalignment using a self-calibration procedure. ${ }^{8}$

The image acquisition was performed at $20 \mathrm{kHz}$ (10000 PIV samples a second) at a resolution of $1024 \mathrm{x} 1024$ pixels. The time between the two laser pulses was set according to the local velocity amplitude and to the laser sheet width (which was set at $2 \mathrm{~mm}$ ), and ranged between 4 and $5 \mu \mathrm{s}$. For each acquisition, 42000 image pairs were acquired. PIV calculations were carried out using a commercial software, and a multi-pass iterative PIV algorithm with deforming interrogation area ${ }^{9}$ to account for the local mean velocity gradients. The PIV interrogation area size was set to $32 \times 32$ pixels for the first pass, decreased at 16x16 pixels for the remaining passes, with an overlap of $50 \%$ between two neighboring interrogation areas. Displacements computed were retained only if the correlation peak-ratio was higher than 1.3. After each pass, a Universal Outlier Detection (UOD) was applied on a $3 \times 3$ vector grid to avoid corrupted data and to enhance the particle motion calculation. Finally, prior to the computation of flow statistics, a 5-sigma filter was applied to remove the remaining outliers which are replaced using the UOD technique.

\section{B. Numerical setup}

To complement the experimental campaign, the jet configuration is investigated with the high-fidelity LES framework developed at Cascade Technologies. ${ }^{10}$ The framework is composed of the pre-processing mesh adaptation tool "Adapt", the compressible flow solver "Charles," and post-processing tools for far-field noise 


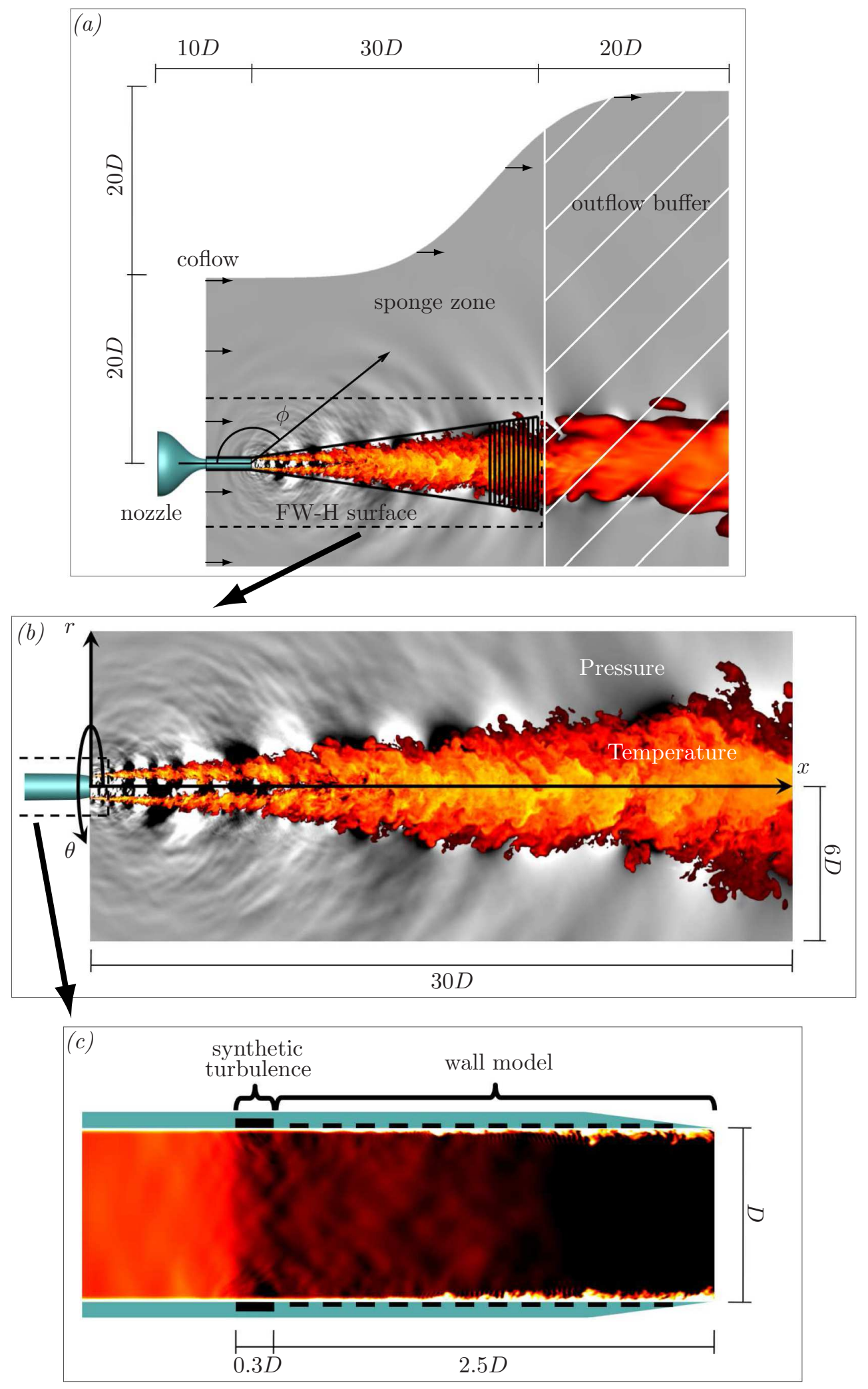

Figure 2. Schematics of the flow configuration and simulation setup: (a) overview of the computational domain; (b) spatial extent of the LES database; (c) modeling inside the nozzle. 
predictions based on an efficient massively-parallel implementation ${ }^{11}$ of the frequency-domain permeable formulation $^{12}$ of the Ffowcs Williams \& Hawkings ${ }^{13}$ (FW-H) equation.

The numerical setup and methodologies are described in more details in Refs. ${ }^{4,5,10}$ and are only briefly summarized here. The LES data uses the following nondimensionalization:

$$
\rho=\frac{\rho^{*}}{\rho_{\infty}^{*}}, \quad p=\frac{p^{*}}{\rho_{\infty}^{*} c_{\infty}^{*}{ }^{2}}, \quad T=\frac{T^{*}}{T_{\infty}^{*}}, \quad u=\frac{u^{*}}{c_{\infty}^{*}}, \quad x=\frac{x^{*}}{D^{*}}, \quad t=\frac{t^{*} c_{\infty}^{*}}{D^{*}}, \quad f=\frac{f^{*} D^{*}}{c_{\infty}^{*}},
$$

where the superscript $*$ refers to the dimensional quantity, and the subscript $\infty$ denotes the ambient (freestream) property. The nondimensionalization is based on the nozzle diameter $D$ and the ambient speed of sound $c_{\infty}=\sqrt{\gamma p_{\infty} / \rho_{\infty}}$, where $\gamma=1.4$. The resulting form of the ideal gas law is $p=\rho T / \gamma$.

The nozzle pressure ratio and nozzle temperature ratio are $N P R=P_{t} / P_{\infty}=1.7$ and $N T R=T_{t} / T_{\infty}=$ 1.15 , respectively, and match the experimental conditions. Here, the subscript $t$ refers to the stagnation (total) property. The jet is isothermal $\left(T_{j} / T_{\infty}=1.0\right)$, and the jet Mach number is $M_{j}=U_{j} / c_{j}=0.9$, where $U_{j}$ is the mean (time-averaged) streamwise jet velocity and the subscript $j$ refer to the jet properties. For both experiment and simulation, the Reynolds number is $R e=\rho U_{j} D / \mu \approx 1 \times 10^{6}$.

The round nozzle geometry (with exit centered at $(0,0,0)$ ) is explicitly included in the axisymmetric computational domain, which extends from approximately $-10 D$ to $50 D$ in the streamwise (x) direction and flares in the radial direction from $20 D$ to $40 D$ (see figure 2(a)). A very slow coflow at Mach number $M_{\infty}=0.009$ is imposed outside the nozzle in the simulation $\left(M_{\infty}=0\right.$ in the experiment), to prevent any spurious recirculation and facilitate flow entrainment. Sponge layers and damping functions are applied to avoid spurious reflections at the boundary of the computational domain. ${ }^{14,15}$ The Vreman ${ }^{16}$ sub-grid model is used to account for the physical effects of the unresolved turbulence on the resolved flow.

As shown in figure $2(c)$ ), synthetic turbulence boundary conditions are used to model the boundary layer trip present in the experiment at $-2.8<x / D<-2.5$ on the internal nozzle surfaces. To properly capture the internal turbulent boundary layers, localized isotropic mesh refinement and wall modeling ${ }^{17-19}$ are applied on the interior surface from the boundary layer trip to the nozzle exit. All the other solid surfaces are treated as no-slip adiabatic wall.

Two grids were generated with the same isotropic near-wall mesh refinement of the boundary layers inside the nozzle: a standard mesh containing approximately 16 million unstructured control volumes (cv), mostly hexahedral, and a refined mesh with 69 million cv, by doubling the resolution in the each refinement zones in the jet plume. Table 1 lists the simulation parameters and settings for the LES runs on the two grids, including the time step $d t$, the total simulation time $t_{\text {sim }}$ for the collection of statistics and data (after the initial transient is removed), and the sampling period $\Delta t$ for the recording for the main LES databases. Note that the sampling period for the recording of the FW-H surface data is $0.25 \Delta t$. For the refined case BL69M_WM_Turb, a subset of the simulation time was also collected at four times the sampling rate used in the main database, to investigate small-scale, high-frequency instabilities of the initial shear-layer.

\begin{tabular}{|l|c|cc|c|c|c|c|c|c|}
\hline Case name & $\begin{array}{c}\text { Mesh } \\
\text { size }\end{array}$ & \multicolumn{2}{|c|}{$\begin{array}{l}\text { Refinement } \\
\text { BL jet }\end{array}$} & $M_{j}$ & $T_{j} / T_{\infty}$ & $R e$ & $d t c_{\infty} / D$ & $\Delta t c_{\infty} / D$ & $t_{\text {sim }} c_{\infty} / D$ \\
\hline \hline BL16M_WM_Turb & $15.9 \times 10^{6}$ & $\times$ & 0.9 & 1.0 & $10^{6}$ & 0.001 & 0.2 & 2000 \\
\hline BL69M_WM_Turb & $69.0 \times 10^{6}$ & $\times$ & $\times$ & 0.9 & 1.0 & $10^{6}$ & 0.0005 & $\begin{array}{c}0.2 \\
0.05\end{array}$ & $\begin{array}{c}1150 \\
500\end{array}$ \\
\hline
\end{tabular}

Table 1. Operating conditions and simulation parameters of the main LES, where $t_{\text {sim }}$ is the simulation time and $\Delta t$ is the sampling period of the database recording.

\section{Far-field acoustic predictions}

The Ffowcs Williams-Hawkings (FW-H) surface used to compute the far-field noise is presented in figure 2(a), along with visualization of the instantaneous temperature and pressure field. In the preliminary study, ${ }^{4,5}$ three conical FW-H surfaces were considered. The surfaces all extend to $x=30 D$, with different spreading rates in the jet plume. As the noise predictions showed nearly identical spectra over the whole frequency range for the three FW-H surfaces, only the results for the intermediate surface are reported in the present 
paper. For the treatment of the FW-H outflow disk, the method of "end-caps" of Shur et al. ${ }^{20}$ is applied for $x>25 D$, where the complex far-field pressure predicted from eleven FW-H surfaces with the same shape but outflow disks at different streamwise locations are phase-averaged.

For far-field noise predictions at a microphone position $\mathbf{x}$, the output of the frequency-domain $\mathrm{FW}-\mathrm{H}$ solver is the complex acoustic pressure $\tilde{p^{\prime}}(\mathbf{x}, f)$ as a function of frequency (i.e., same nondimensionalization than LES data). The narrowband (one-sided) Power Spectral Density $(P S D)$ is then calculated as

$$
P S D(\mathbf{x}, f)=\left(\frac{2 \tilde{p^{\prime}}(\mathbf{x}, f){\tilde{p^{\prime}}}^{\dagger}(\mathbf{x}, f)}{f_{\min }}\right),
$$

and the Overall Sound Pressure Level $(O A S P L)$ is computed as

$$
O A S P L(\mathbf{x})=\left(\sum_{f_{\min }}^{f_{\max }} 2 \tilde{p^{\prime}}(\mathbf{x}, f){\tilde{p^{\prime}}}^{\dagger}(\mathbf{x}, f)\right)
$$

where the superscript $\dagger$ denotes the complex conjugate. The parameters $f_{\max }=0.5 D /\left(d t c_{\infty}\right)$ and $f_{\min }=$ $D /\left(t_{\text {sim }} c_{\infty}\right)$ are the maximum frequency (i.e., Nyquist limit) and minimum frequency (i.e., the narrowband width) accessible by the post-processing. In the present work, the noise is computed on a cylindrical microphone array of constant radius $14.3 \mathrm{D}$ from the jet axis, with 18 equally-spaced microphones in the azimuthal direction matching the experiment (see figure 1(b)), and on a polar microphone array at a constant distance $50 D$ from the nozzle exit, with 36 equally-spaced microphones in the azimuthal direction.

The PSD is reported in $\mathrm{dB} / \mathrm{St}$, versus frequency in Strouhal $S t=f D / U_{j}$, with respect to the dimensional reference pressure $P_{r e f}^{*}=20 \mu \mathrm{Pa}$, following the same non-dimensionalization than the experiment (see Appendix of Ref. 5). To compensate for the relatively short time signal in simulation compared to experiment and to allow a straight comparison between the data sets with different total time, bin-averaged PSD is computed, with bin size $\Delta S t=0.05$, for all the numerical data.

\section{Interpolation of the LES data onto structured grids}

To facilitate postprocessing and analysis, the LES data is interpolated from the original unstructured LES grid onto structured cylindrical grids in the jet plume and in the nozzle pipe. These structured cylindrical grids were originally designed for the case BL16M_WM_Turb, such that the resolution approximately corresponds to the underlying LES resolution. In the figures 3 and 4, the point clouds are the resolution distributions of the unstructured LES grid (in the subset of the domain $y / D \geq 0, z / D \geq 0$ ) and the black curves are the chosen resolutions for the structured grids.

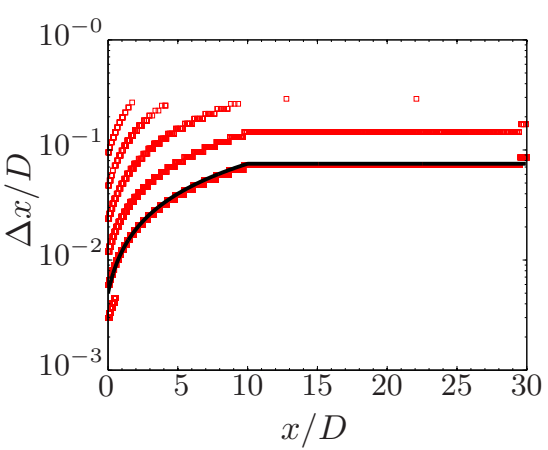

(a) streamwise resolution

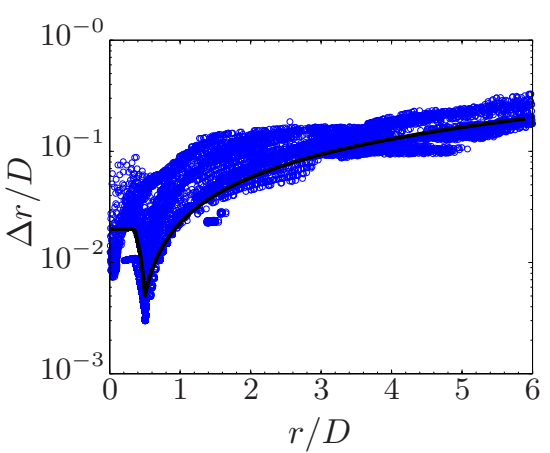

(b) radial resolution

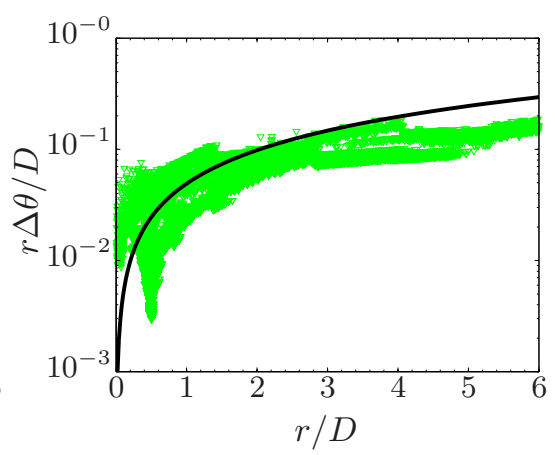

(c) azimuthal resolution

Figure 3. Representation (in logarithmic scale) of the mesh resolution in the jet plume: (colored symbols) unstructured LES grid for case BL16M_WM_Turb; (black lines) structured cylindrical grid for the database.

For the jet plume, the three-dimensional cylindrical grid extents to $0 \leq x / D \leq 30,0 \leq r / D \leq 6$, with $\left(n_{x}, n_{r}, n_{\theta}\right)=(626,138,128)$, where $n_{x}, n_{r}$ and $n_{\theta}$ are the number of points in the streamwise, radial and azimuthal direction, respectively (see figure 2(b)). For the nozzle pipe, the cylindrical grid extents to 


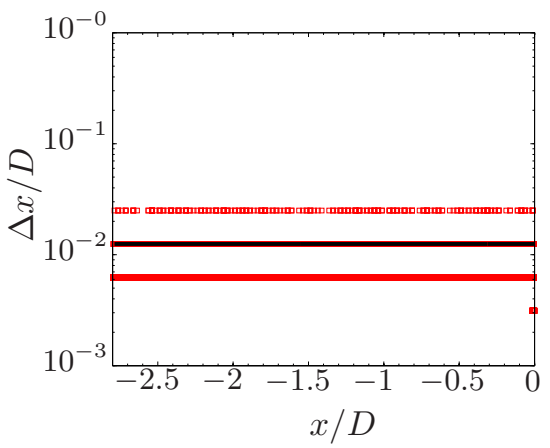

(a) streamwise resolution

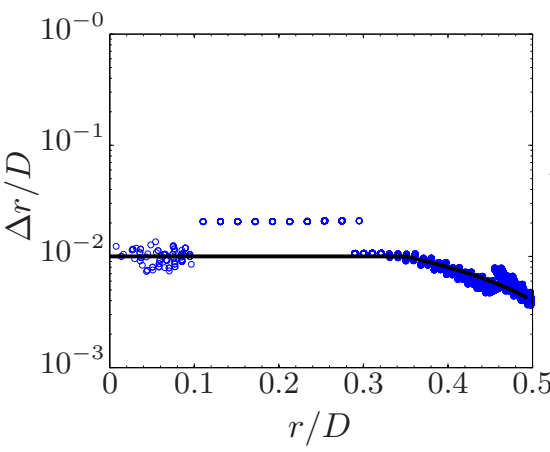

(b) radial resolution

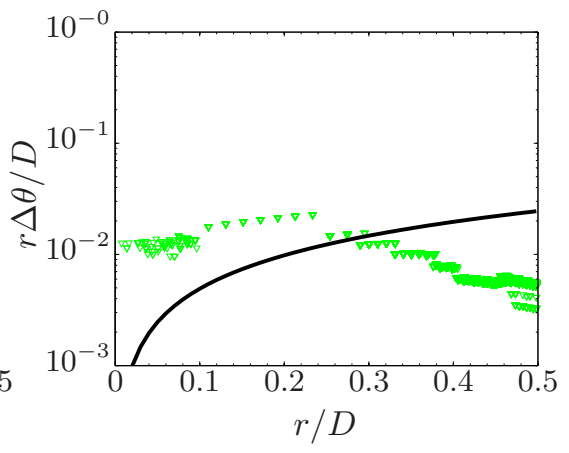

(c) azimuthal resolution

Figure 4. Representation (in logarithmic scale) of the mesh resolution in the nozzle pipe: (colored symbols) unstructured LES grid for case BL16M_WM_Turb; (black lines) structured cylindrical grid for the database.

$-2.8 \leq x / D \leq 0,0 \leq r / D \leq 0.5$, with $\left(n_{x}, n_{r}, n_{\theta}\right)=(225,58,128)$. For both grids, the points are equallyspaced in the azimuthal direction to enable simple azimuthal decomposition in Fourier space. The point positions $x, y, z$ and the instantaneous value of the primitives variables density $\rho$, velocity (in cartesian coordinate) $u, v, w$ and pressure $p-1 / \gamma$ are saved as single-precision ("float") numbers to reduce disk space.

Thought the underlying LES resolution is essentially doubled in the jet plume for the refined case BL69M_WM_Turb, the main database was extracted onto the same structured cylindrical grids, to simplify the analyses and comparisons. In addition, an secondary database was interpolated in the near-nozzle exit region for the subset of the simulation time collected at the higher sampling rate (see table 1). The two near-nozzle cylindrical grids correspond to $0 \leq x / D \leq 5,0 \leq r / D \leq 1$, with $\left(n_{x}, n_{r}, n_{\theta}\right)=(556,93,128)$, and $0.5 \leq x / D \leq 0,0 \leq r / D \leq 0.5$, with $\left(n_{x}, n_{r}, n_{\theta}\right)=(49,61,128)$. The total size of the resulting database is approximately $2.6 \mathrm{~Tb}$ for BL16M_WM_Turb and $2.9 \mathrm{~Tb}$ for BL69M_WM_Turb.

\section{Summary of the LES and experimental results}

\section{A. Flow field}

The nozzle exit velocity profiles are presented in figure 5. Both experimental hot-wire measurements and LES results are reported at the same location just downstream of the nozzle exit, at $x / D=0.04$. Note that the very slow coflow $M_{\infty}=0.009$ imposed in the simulation outside the nozzle is the main cause of the slight mismatch in mean velocity for $y / D<-0.5$.

As previously discussed, both LES have the same adapted mesh inside the nozzle and the same synthetic turbulence and wall modeling applied to the nozzle internal walls, to reproduce the effects of the azimuthally homogeneous carborundum strip present in the experiment upstream in the pipe. This leads to the same turbulent profiles for the nozzle-exit boundary layer, in good agreement with the measurements. The only noticeable difference is for the maximum RMS levels at $r / D=0.5$. Here, the additional resolution in the jet plume for the refined case BL69M_WM_Turb is better suited to resolve the sharp peak of the RMS levels at the lipline. That peak is likely missed in the measurement because of limited spatial resolution.

A new campaign of PIV measurements was conducted in 2015 at the "Bruit et Vent" jet-noise facility at the CEAT, PPRIME Institute, Poitiers, France. This was done to reduce lens distortion and correct a minor misalignment along the jet axis in the previous PIV data. ${ }^{5}$ The new measurements have however a slightly smaller signal to noise ratio, in particular in the near nozzle exit region. Figure 6 shows the comparisons of the centerline and lipline profiles for streamwise velocity statistics in the jet plume between PIV and LES for the two different grid resolutions.

Overall, there is a good agreement between the experimental and numerical results, for both mean and RMS streamwise velocity. The length of the potential core, as well as the location and amplitude of the peak RMS fluctuation along the centerline, are well predicted by the simulations. Along the lipline, The LES results from the two mesh resolutions are essentially identical up to $x / D \approx 10$. Further downstream of the potential core, the refined case tends to display slightly higher mean and RMS values than the standard case, as the increase in resolution in that region leads to prediction improvements of the turbulent mixing. 


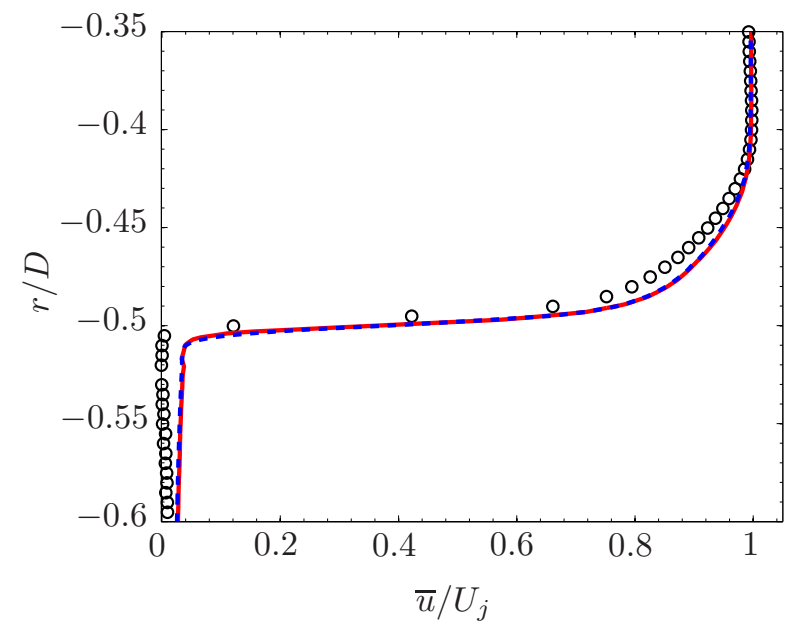

(a) Time-averaged streamwise velocity

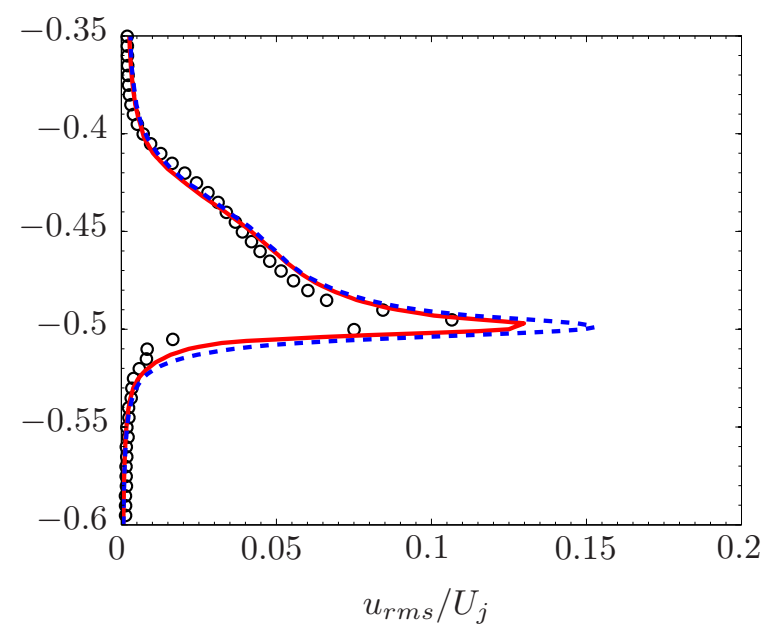

(b) RMS of streamwise velocity

Figure 5. Nozzle-exit boundary layer profiles from experiment ( ० ) and LES cases BL16M_WM_Turb ( - ) and BL69M_WM_Turb (---- ).

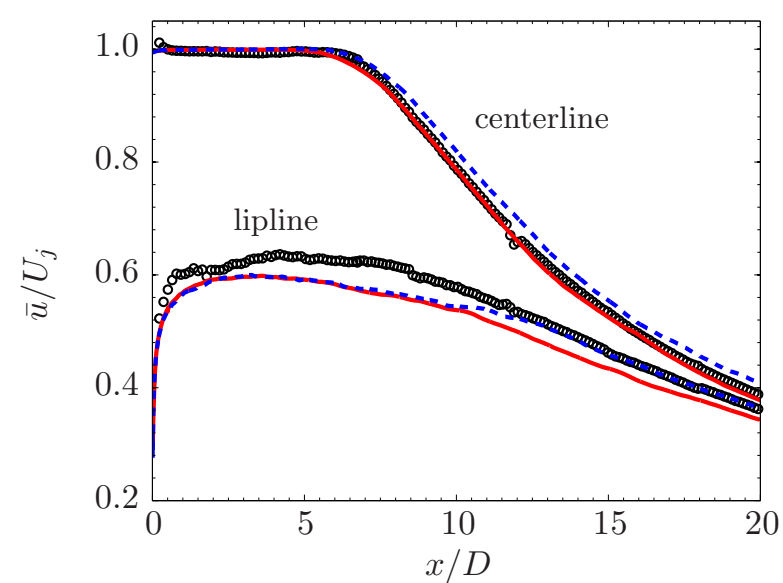

(a) Time-averaged streamwise velocity

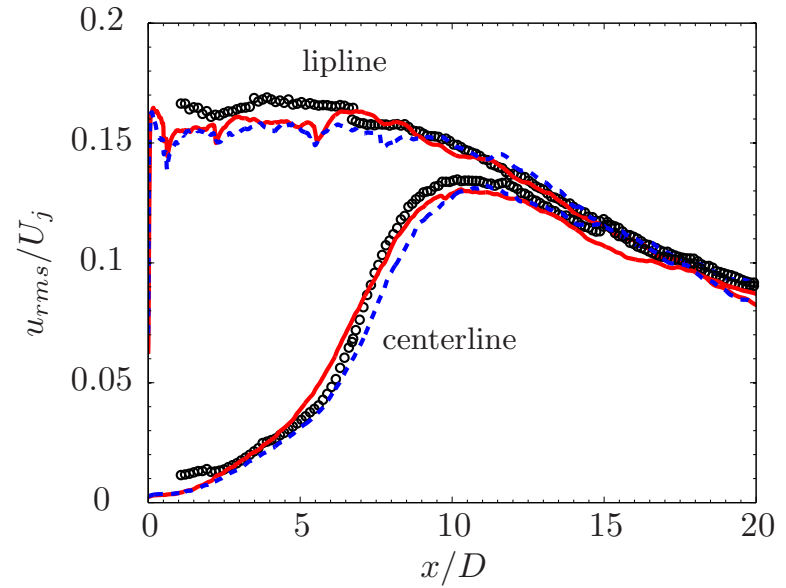

(b) RMS of streamwise velocity

Figure 6. Centerline and lipline profiles of the streamwise velocity statistics from experiment $(\circ)$ and LES cases BL16M_WM_Turb ( $\longrightarrow$ ) and BL69M_WM_Turb (---- ).

\section{B. Acoustic field}

For all the near-field and far-field microphones, the complete comparison with the FW-H predictions is presented in figure 7 , where $\phi$ is the jet inlet angle. Figure 8 shows the corresponding overall sound pressure level directivity (OASPL) in $\mathrm{dB}$, where the frequency range considered for the integration is $0.05 \leq S t \leq 3$. Note that the microphones on the cylindrical array are not at a constant distance for the nozzle exit, which accounts in part for the shape difference in the directivity curves. In all these figures, the spectra from the azimuthal microphones are ensemble-averaged.

Overall, the agreement between experiment and simulation is excellent. For most inlet angles and relevant frequencies, there is little differences between the two LES results and the numerical predictions are typically within $0.5 \mathrm{~dB}$ of the measurements. Aside from the slight variations in the very low frequencies due to temporal convergence, the main differences between the LES results from the two mesh resolutions are observed in the grid cut-off frequency for the high inlet angles $\phi \geq 150^{\circ}$ : at these angles, the limit frequency is about $S t \approx 2$ for the standard case BL16M_WM_Turb and $S t \approx 4$ for the refined case BL69M_WM_Turb with double the resolution in the jet plume. Here, it is important to note that these discrepancies are outside of the main frequency range of interest and with levels 25 to $30 \mathrm{~dB}$ lower that the peak radiated 


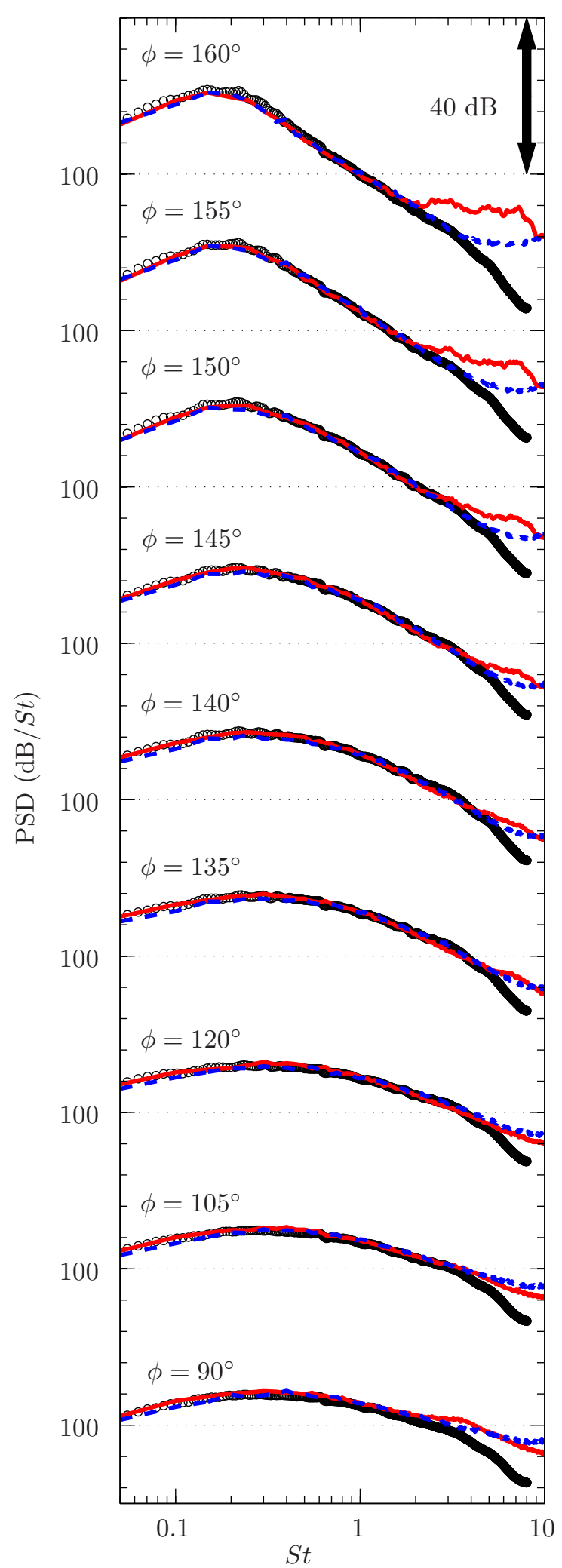

(a) Cylindrical array of radius $r=14.3 \mathrm{D}$

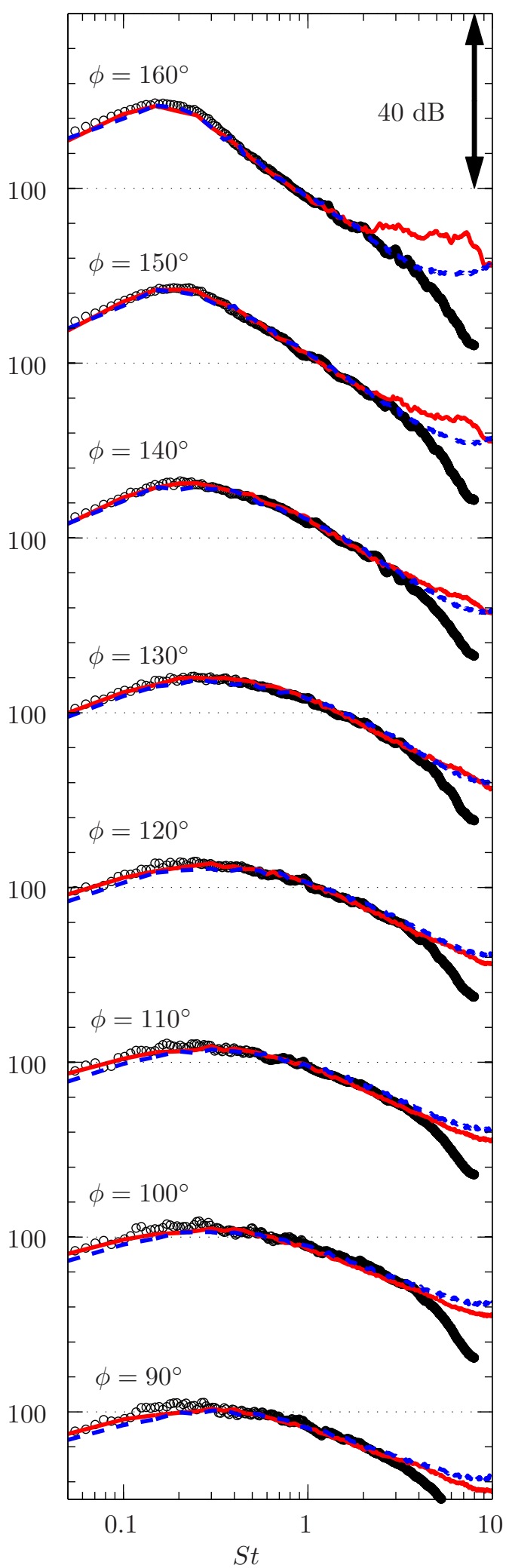

(b) Far-field array at constant distance $50 D$

Figure 7. Comparison of the noise spectra from experiment $(\circ)$ and LES cases BL16M_WM_Turb ( - $($ ) and BL69M_WM_Turb ( ---- ) for all the experimental microphones. 


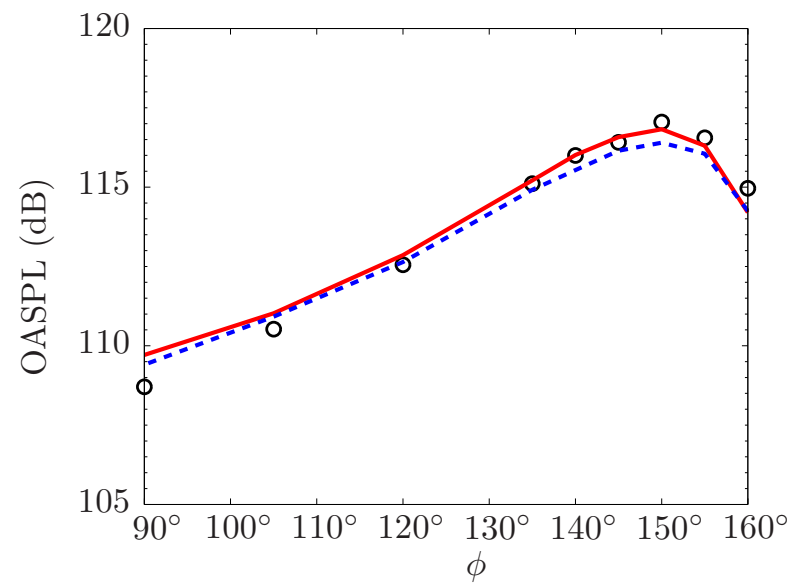

(a) Cylindrical array of radius $r=14.3 D$

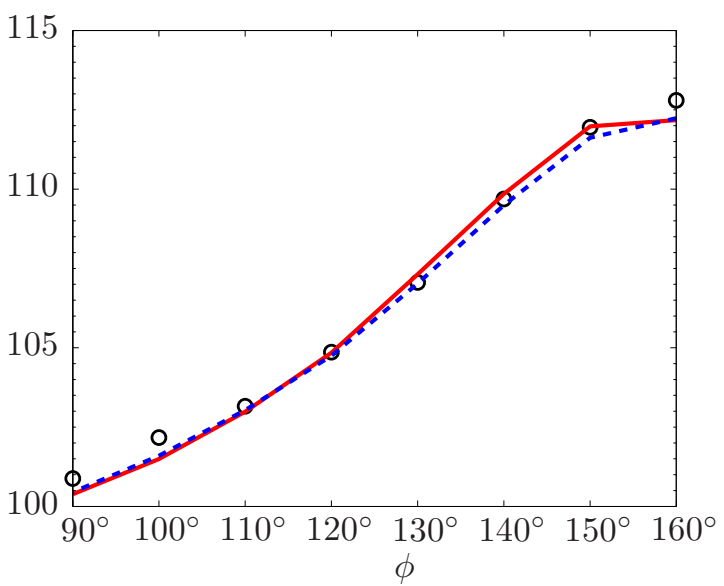

(b) Far-field array at constant distance $50 D$

Figure 8. Comparison of the the overall sound pressure level directivity from experiment $(\circ)$ and LES cases BL16M_WM_Turb ( — ) and BL69M_WM_Turb (---- ).

noise, such that they do not significantly impact the predictive capabilities nor the use of the database for noise modeling.

\section{Postprocessing and analysis}

\section{A. Preliminary results on noise azimuthal mode decomposition}

Previous experimental ${ }^{21-24}$ and numerical ${ }^{25}$ jet studies have suggested that low-frequency noise (i.e., Strouhal number $S t<1)$ may be decomposed into just 3 Fourier azimuthal mode $m=0,1$ and 2 . This feature has important implications towards modeling of noise-source mechanism, as well as noise reduction strategies.

As a complement to experimental studies (typically limited in the past to a few microphones), the LES database provides access to the complete flow field for in-depth probing of the physics of jet noise production. As a first step and to leverage the available measurements, the azimuthal decomposition was performed using the FW-H predictions from 18 microphones evenly-spaced in the azimuthal direction, on the same cylindrical array of radius $14.3 D$ as the experiment (see Figure $1(b)$ ). The output is the complex acoustic pressure as a function of frequency and azimuthal mode $m$ at each jet inlet angle on the array.

The analysis was performed independently for both the experimental and LES data and the results are reported for a few representative inlet angles in figure 9. In this figure, the total noise spectra from experiment (black circle) and LES (red line) is the same data reported in figure 7 $(a)$. For individual modes, the agreement between measurement and prediction is again excellent, in particular towards the peak radiation angles $\phi=150^{\circ}-160^{\circ}$. At these angles, the $m=0$ azimuthal mode is clearly dominant, followed by mode $m=1$ and then $m=2$, which is confirmed in figure 10 by the OASPL curves, computed for the frequency range $0.05 \leq S t \leq 3$. In this figure, the total OASPL is compared to the OASPL calculated with only selected azimuthal modes retained for the pressure, namely either mode $m=0$ only, modes $m=0 \& 1$, or modes $m=0,1 \& 2$. At $\phi=160^{\circ}$, the $m=0$ azimuthal mode contributes to $86 \%$ of the total acoustic energy, and this value goes to more than $99.2 \%$ when the first 3 modes are considered. Over all angles, the first 3 Fourier azimuthal modes of the LES data recover more than $65 \%$ of the total acoustic energy, which means that a prediction based on these 3 dominant modes would be within $1.9 \mathrm{~dB}$ of the total value. These results are all consistent with the experimental trends previously reported in the literature.

At the lower inlet angles $\phi<135^{\circ}$, the mode order (in terms of importance) tends to be reversed, with mode $m=2$ more energetic than $m=1$ and then $m=0$, but the difference in contribution between the three modes is less pronounced. This can be clearly seen in figure 11, where the spectra from figure 9 are extracted at specific low frequency of interest $S t=0.1,0.2$ and 0.3 , and are plotted as a function of the inlet angle. The trend is consistent across these Strouhal numbers corresponding to the frequency range of the main wave packet radiation, with again excellent agreement between experimental measurements and numerical predictions. 


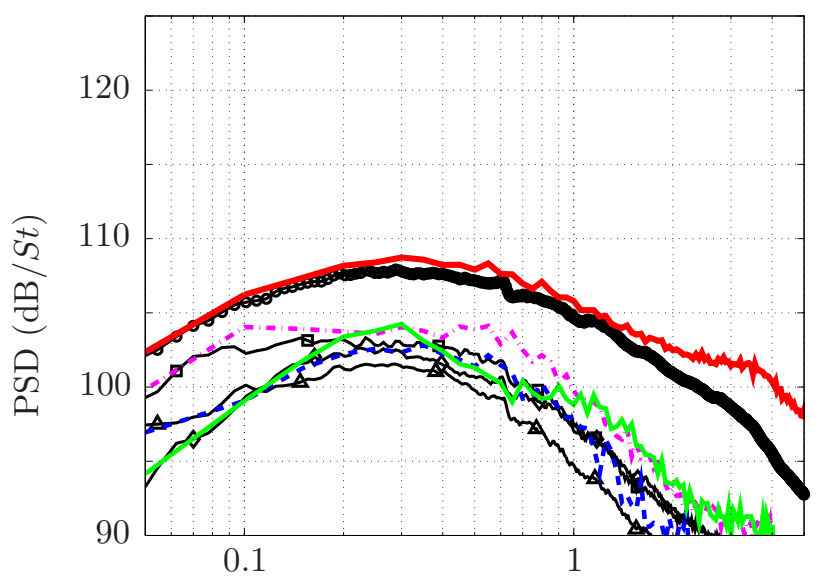

(a) $\phi=90^{\circ}$

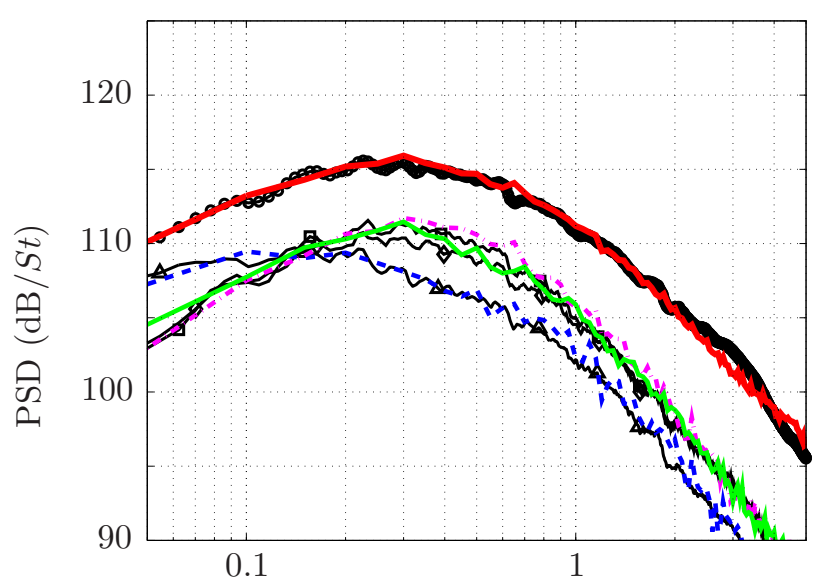

(c) $\phi=135^{\circ}$

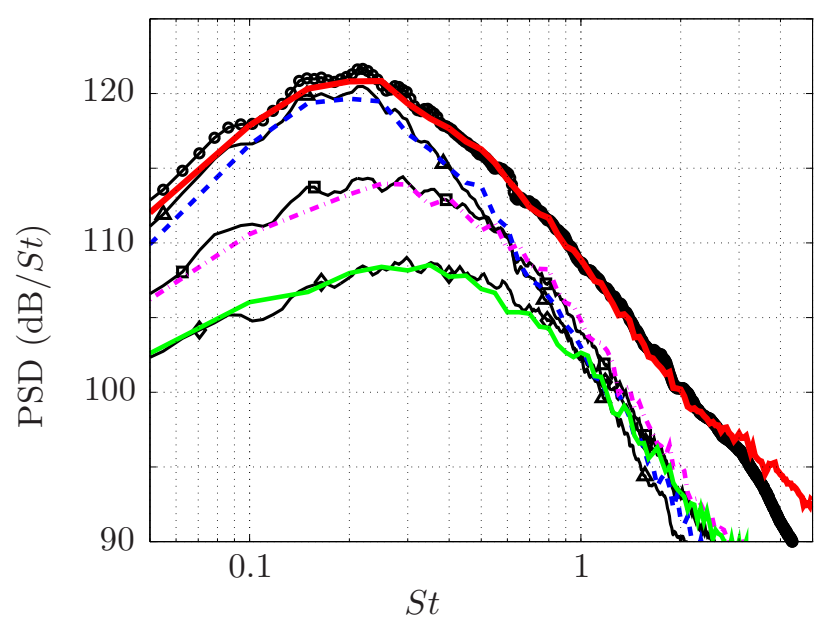

(e) $\phi=150^{\circ}$

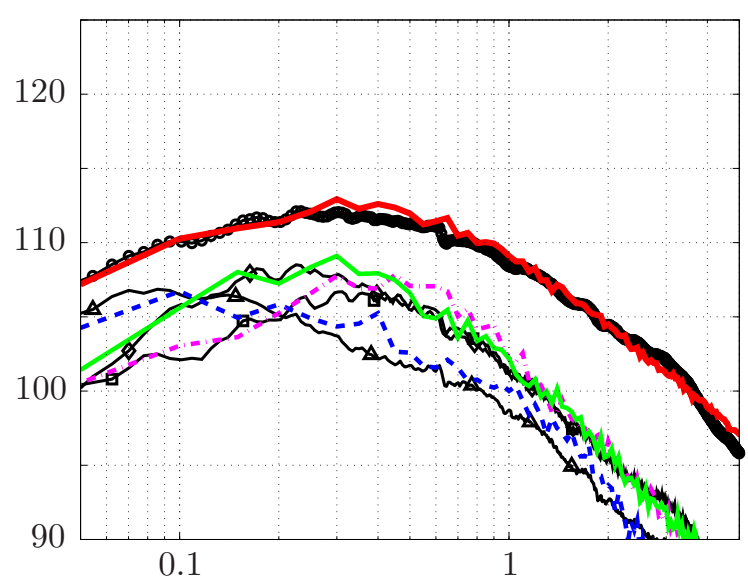

(b) $\phi=120^{\circ}$

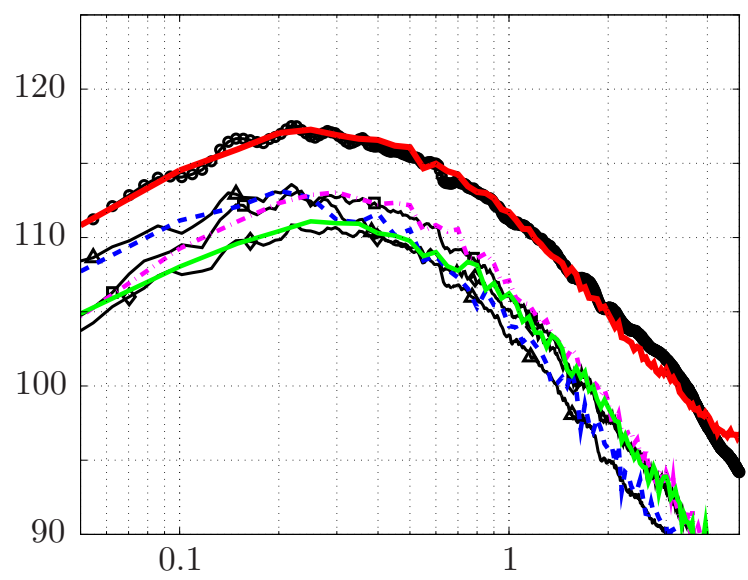

(d) $\phi=140^{\circ}$

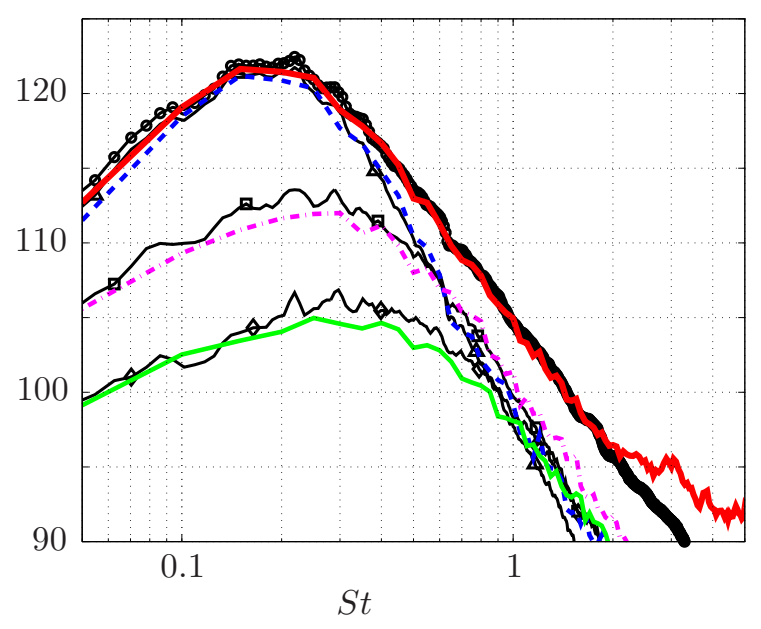

$(f) \phi=155^{\circ}$

Figure 9. Azimuthal decomposition of the radiated noise at specific jet inlet angles for the experimental data (black lines and symbols) and LES data from case BL16M-WM_Turb (color lines): ( o, modes $) ;(\triangle,----)$ mode $m=0 ;(\square,---)$ mode $m=1 ;(\diamond,-$ ) mode $m=2$. 
The azimuthal analysis was repeated for the LES noise data on a 36-microphone far-field array at constant distance $50 D$. There is no experimental equivalent here since the measurements were done with only a single microphone. Overall, the same trends were observed, which demonstrate that the 18-microphone array is sufficient to capture the dominant azimuthal components of the radiated noise.

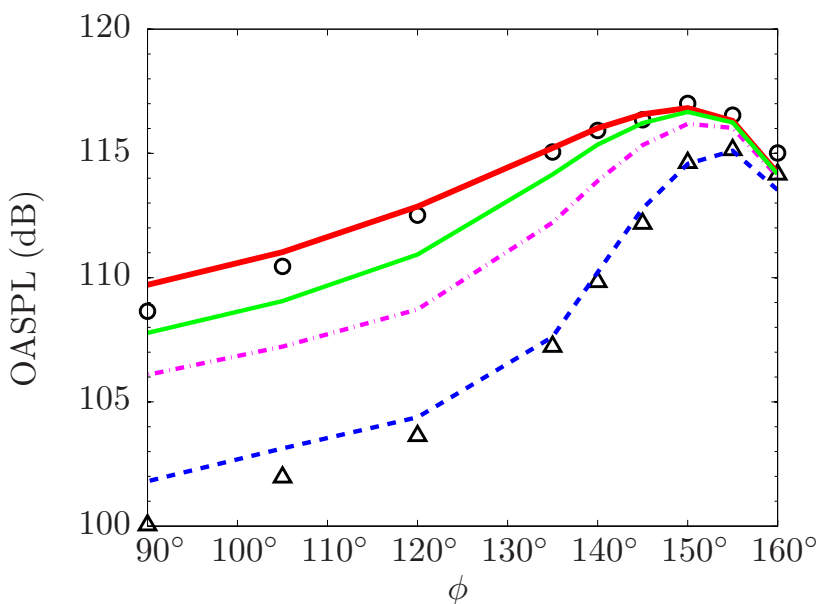

(a) Overall Sound Pressure Level

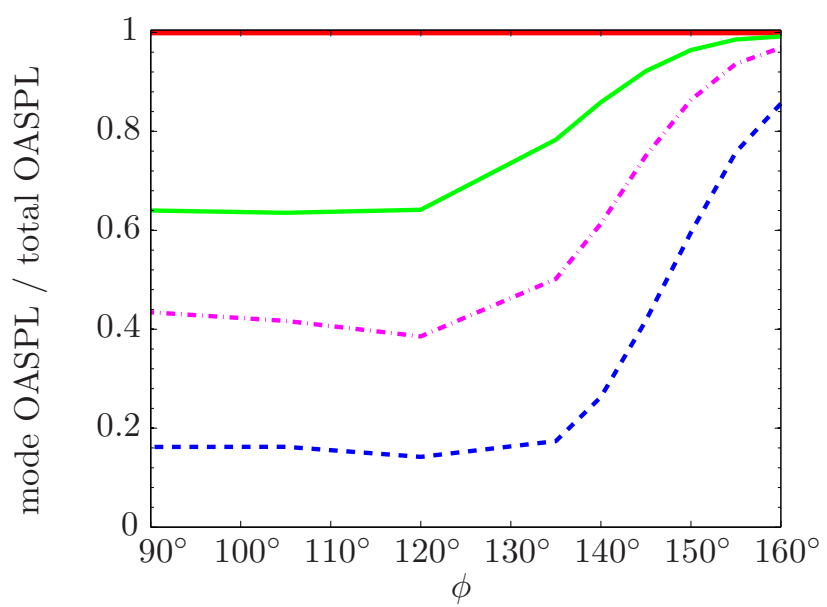

(b) Mode contributions

Figure 10. Azimuthal analysis of the radiated noise OASPL for the experimental data (black symbols) and LES data from case BL16M_WM-Turb (color lines):( $\circ, \underline{-}$ ) total (i.e., all modes); ( $\triangle,---$ ) mode $m=0 ;(--$ ) modes $m=0 \& 1 ;(-)$ mode $m=0,1 \& 2$.

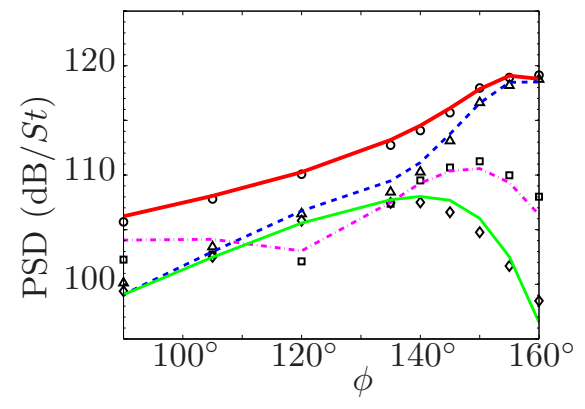

(a) $S t=0.1$

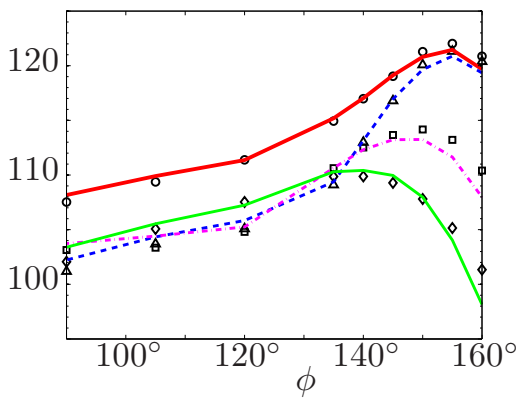

(b) $S t=0.2$

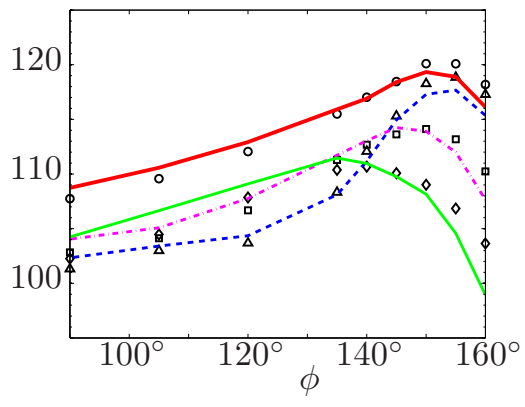

(c) $S t=0.3$

Figure 11. Azimuthal decomposition for the radiated noise at specific frequencies for the experimental data (black symbols) and LES data from case BL16M_WM_Turb (color lines): ( o, _ - ) total (i.e., all modes); $(\triangle,----)$ mode $m=0 ;(\square,-\cdot-)$ mode $m=1 ;(\diamond,-)$ mode $m=2$.

\section{B. Preliminary results on noise temporal intermittency}

Another important characteristic of the far-field noise is its temporal intermittency. As previously reported, ${ }^{23,25,26}$ the peak radiated noise around $S t \approx 0.2$ has been observed to recur in temporally-localized bursts. In general, this behavior can be challenging to detect and quantify in simulations because the total time duration of the recorded data is not sufficient, typically several orders of magnitude shorter than in experiments. Here, the long LES database generated during this project enables some preliminary analysis of the far-field noise temporal intermittency.

As reported in table 1, the total simulation time (after the initial transient was removed) for the case BL16M_WM_Turb is $t_{\text {sim }} c_{\infty} / D=2000$. While the far-field noise spectra reported in the previous sections was computed using the full 2000 time units, the present analysis uses blocks of 250 times units of FW-H surface data as input to the FW-H solver. With $75 \%$ overlap, the total simulation time generates 30 blocks, with minimum frequency accessible in each block $S t_{\min }=0.00444$, compared to $S t_{\min }=D /\left(t_{\operatorname{sim}} U_{j}\right)=0.00056$ for the full data. Following the same procedure applied to the full data, the narrowband spectra for each 
block is then bin-averaged with bin size $\Delta S t=0.05$. Here, the choice of 250 acoustic time units for the block size ensures that the averaging is done with at least 11 periods at $S t=0.05$ (i.e., the lowest frequency considered). This value is also representative of the typical run time in LES of high-speed jets ${ }^{11,20,27-30}$

Figure 12 shows the individual block spectra (blue square symbols) superposed on the data presented in figure $7(b)$ for the far-field array at constant distance $50 D$. As expected, the spectra ensemble-averaged over all blocks is essentially identical to the spectra from the FW-H calculation on the full signal (red curve) and is therefore not shown on the figure for clarity. While there is some scattering for all angles and frequencies, the block-to-block variation is more pronounced for the low frequencies $S t=0.1$ to 0.3 at the peak radiation angle of $\phi=150^{\circ}$. At these frequencies and angles, the predicted noise levels in some of the time blocks can be as much as 3 or $4 \mathrm{~dB}$ higher (or lower) than the long-time average.

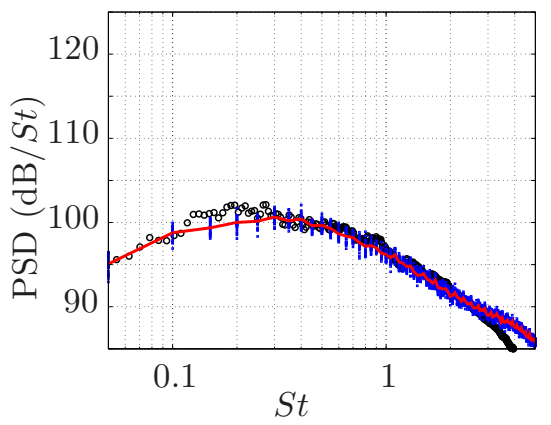

(a) $\phi=90^{\circ}$

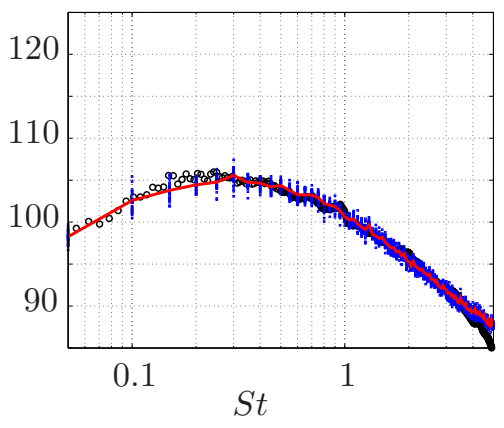

(b) $\phi=120^{\circ}$

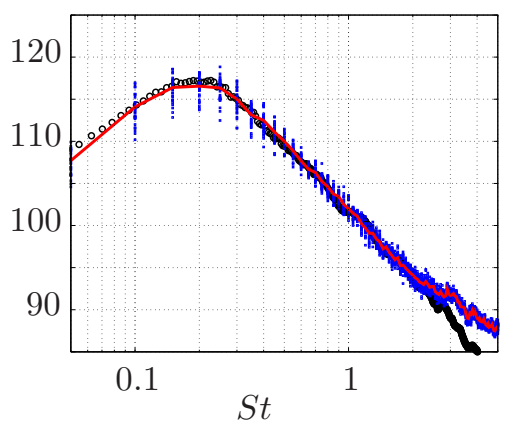

(c) $\phi=150^{\circ}$

Figure 12. Comparison of the noise spectra from experiment ( $\circ$ ) and LES case BL16M_WM_Turb predicted using the full 2000 time units of input data ( - ) , and using blocks of 250 time units with $75 \%$ overlap $(\square)$

To better quantify this temporal intermittency, block predictions are analyzed in terms of probability distribution in figure 13. The analyses focuses on the low frequencies $S t=0.1$ to 0.35 for two representative jet inlet angles $\phi=90^{\circ}$ and $150^{\circ}$. The spectra from the different time blocks are sorted in discrete bins of $1 \mathrm{~dB}$ width centered around the mean spectra predicted using the full 2000 time units of input data (red line). The resulting block counts are then normalized by the total number of blocks, to display the discrete probability distribution of the block PSD prediction in $\mathrm{dB}$ (blue bars).

At $\phi=90^{\circ}$, the different time blocks do not display significant variations, which leads to a probability distribution with a narrow head centered on the mean and a small support (i.e., 3-4 bins): the predictions are within $\pm 0.5 \mathrm{~dB}$ of the mean PSD for 55 to $60 \%$ of the time blocks over that frequency range, and these number increases to 75 to $85 \%$ for an interval of $\pm 1 \mathrm{~dB}$ about the mean. Here, these uncertainties are likely to be related to the limited temporal convergence of the LES data in each individual block rather than temporal intermittency in the jet. Overall, the results do not feature clear asymmetry or skewness, and are reminiscent of normal (Gaussian) distribution.

In contrast, temporal intermittency is clearly observed at $\phi=150^{\circ}$ for the $S t=0.1$ to 0.35 frequency range: the probability distribution has a larger support (i.e., 6-8 bins) and a wider head with the highest value not necessarily centered on the mean PSD. In average, only $26 \%$ of the time blocks yield prediction within $\pm 0.5 \mathrm{~dB}$ of the mean PSD, and $50 \%$ within $\pm 1 \mathrm{~dB}$. Again, the results do not display obvious asymmetry or skewness toward particularly loud (or quiet) recurrent events. Therefore, such distributions tend to suggest the presence of temporally-localized bursts of increased noise alternating with periods of decreased noise at the peak radiation angles and frequencies.

Here, it is important to note that the analysis and probability distributions are based on the limited sample of 30 time blocks available in the LES data. The procedure will be reproduced on the much longer sample of experimental data to confirm and further clarify the present findings. That being said, the main benefit of the numerical database is that it provides a direct access to the corresponding transient flow field that generated a particularly loud (or quiet) burst of radiated noise. 

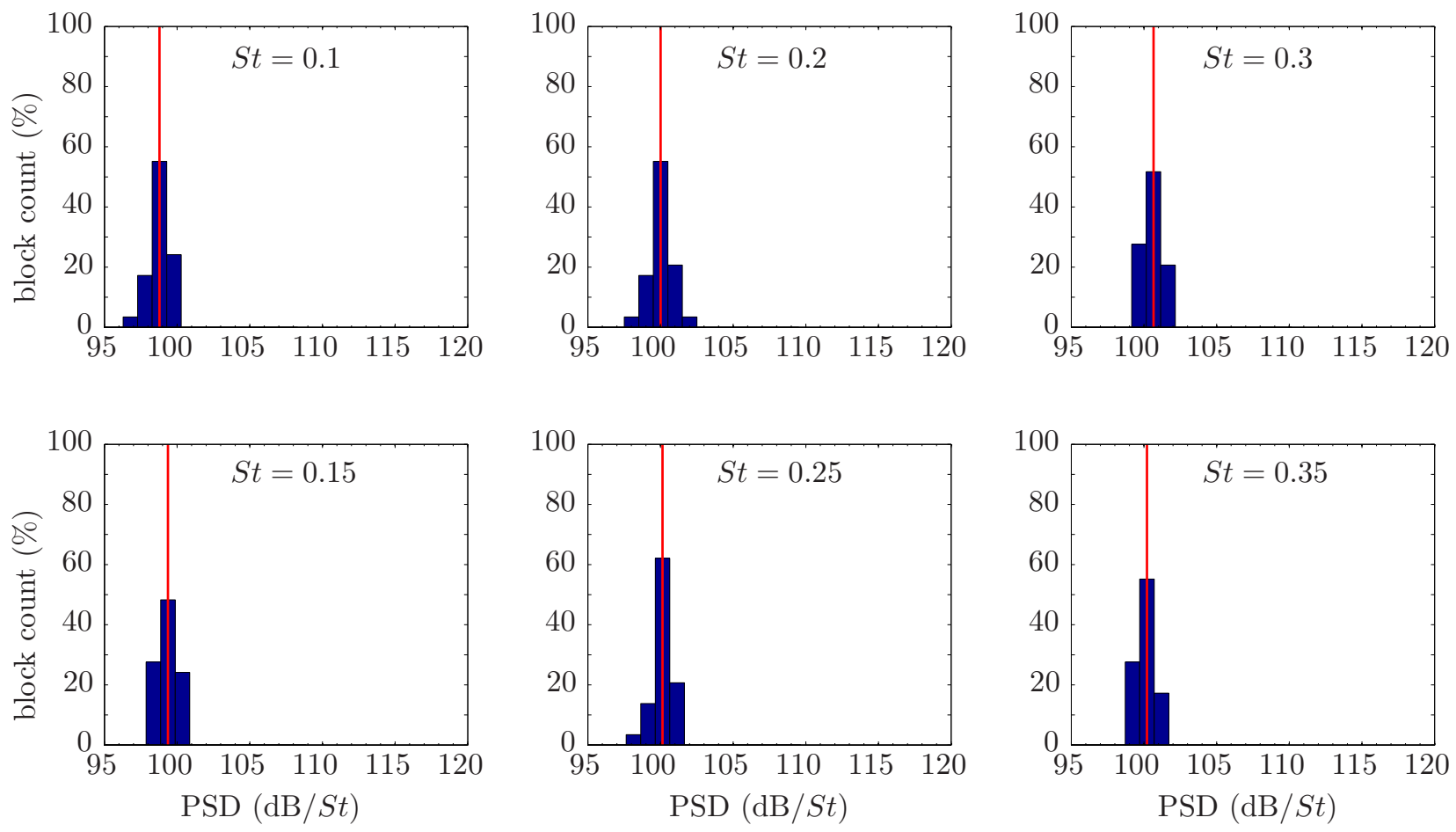

(a) $\phi=90^{\circ}$
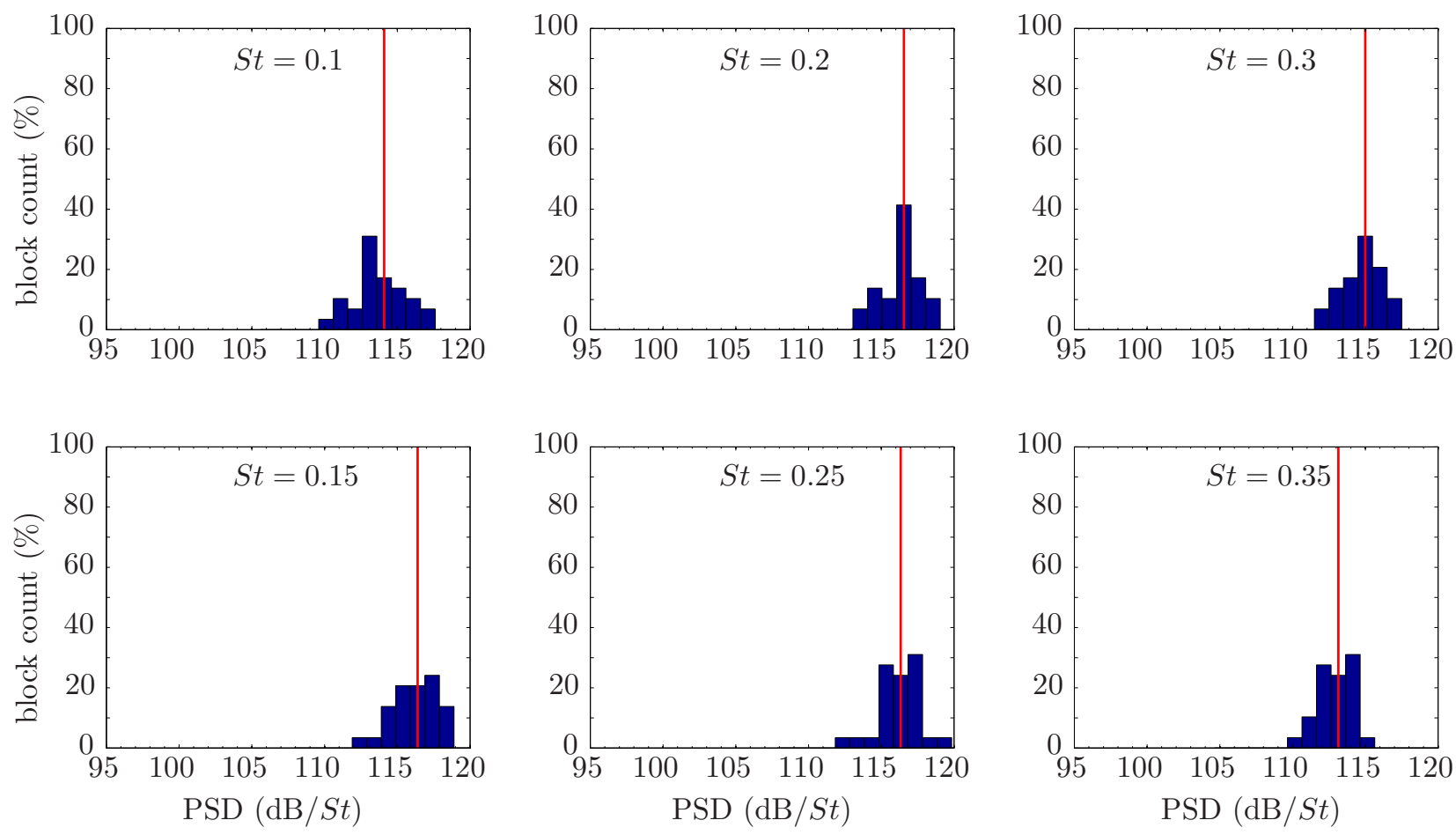

(b) $\phi=150^{\circ}$

Figure 13. Discrete probability distribution of the spectra predicted using blocks of 250 time units with $75 \%$ overlap (blue bars) at frequencies $S t=0.1$ to 0.35 for LES case BL16M_WM_Turb. The mean spectra predicted using the full 2000 time units of input data is also shown ( - ). 


\section{Conclusion and outlook}

Large eddy simulations and experimental measurements of an isothermal Mach 0.9 turbulent jet (Reynolds number $R e \approx 10^{6}$ ) issued from contoured convergent-straight nozzle are performed to generate an extensive database for noise modeling and wavepacket analysis. For the LES, two grids with different mesh resolutions in the jet plume are considered. Both cases have localized adaptive mesh refinement inside the nozzle, as well as synthetic turbulence and wall modeling applied on the nozzle internal surfaces to ensure a fully turbulent jet. With this approach, the nozzle-exit boundary layers conditions match the turbulent profiles from the experiments, and the far-field noise predictions are within $0.5 \mathrm{~dB}$ of the microphone data for all angles and most relevant frequencies.

For both LES cases, long transient flow databases are collected and interpolated from the original unstructured LES grid onto structured cylindrical grids to facilitate postprocessing and analysis. Details of the LES database and validation with experimental measurements are presented, as well as preliminary results on the radiated noise azimuthal decomposition and temporal intermittency. The azimuthal analysis shows that the axisymmetric mode $m=0$ is dominant at the downstream angles $\phi=150^{\circ}$ to $160^{\circ}$ for the main frequency range $0.05 \leq S t \leq 0.5$. At these angles, the first 3 Fourier azimuthal modes of the LES data recover more that $97 \%$ of the total acoustic energy and more than $65 \%$ (i.e., error less than $2 \mathrm{~dB}$ error) over all jet angles. The temporal analysis confirms the presence of temporally-localized bursts of noise in the low-frequency radiated sound, in particular at the peak radiation angle. For the present case, variation in the PSD levels up to 3 or $4 \mathrm{~dB}$ higher (or lower) than the long-time average are observed for several time segments of the long LES database. Further analysis is ongoing to connect this noise intermittency with wavepacket radiation and jittering. While the degree of intermittency may depend on nozzle design, operating conditions, Mach number and other details of the flow, this is an important feature that any numerical or experimental investigation needs to be aware of, and account for, to the extent possible.

As a next step toward better understanding of turbulent jet noise, the database are currently used for reduced-order models ${ }^{31}$ and wavepacket analysis. ${ }^{32-34}$ In particular, the LES data uncovered a novel class of resonant acoustic modes that are trapped within the potential core of the jet. The modes are the topic of several studies focusing on modeling, ${ }^{35}$ global modes analysis ${ }^{36}$ and experimental measurements. ${ }^{37}$

\section{Acknowledgments}

The LES studies are supported by NAVAIR SBIR project, under the supervision of Dr. John T. Spyropoulos. The main calculations were carried out on CRAY XE6 machines at DoD supercomputer facilities in ERDC DSRC. P. Jordan acknowledges the support of the French National Research Agency (ANR) through the project COOLJAZZ.

\section{References}

${ }^{1}$ Jordan, P. and Colonius, T., "Wave Packets and Turbulent Jet Noise," Ann. Rev. Fluid Mech., Vol. 45, 2013, pp. 173-95.

${ }^{2}$ Mollo-Christensen, E., "Jet noise and shear flow instability seen from an experimenter's viewpoint," J. Appl. Mech., Vol. 34, 1967, pp. 1-7.

${ }^{3}$ Crow, S. C. and Champagne, F. H., "Orderly structure in jet turbulence," J. Fluid Mech., Vol. 48, 1971, pp. $547-591$.

${ }^{4}$ Brès, G. A., Jordan, P., Colonius, T., Le Rallic, M., Jaunet, V., and Lele, S. K., "Large eddy simulation of a Mach 0.9 turbulent jet," Proceedings of the Summer Program, Center for Turbulence Research, Stanford University, 2014.

${ }^{5}$ Brès, G. A., Jaunet, V., Le Rallic, M., Jordan, P., Colonius, T., and Lele, S. K., "Large eddy simulation for jet noise: the importance of getting the boundary layer right," AIAA paper 2015-2535, 2015.

${ }^{6}$ Brès, G. A., Ham, F. E., Nichols, J. W., and Lele, S. K., "Nozzle Wall Modeling in Unstructured Large Eddy Simulations for Hot Supersonic Jet Predictions," AIAA paper 2013-2142, 2013.

${ }^{7}$ Brès, G. A., Bose, S. T., Ham, F. E., and Lele, S. K., "Unstructured Large Eddy Simulations for Nozzle Interior Flow Modeling and Jet Noise Predictions," AIAA paper 2014-2601, 2014.

${ }^{8}$ Wieneke, B., "Stereo-PIV using self-calibration on particle images," Experiments in Fluids, Vol. 39, 2005, pp. 267-280.

${ }^{9}$ Scarano, F., "Iterative image deformation methods in PIV," Meas. Sci. Tech., Vol. 13, 2002.

${ }^{10}$ Brès, G. A., Ham, F. E., Nichols, J. W., and Lele, S. K., "Unstructured Large Eddy Simulations of Supersonic Jets," Under review in AIAA J., 2016.

${ }^{11}$ Brès, G. A., Nichols, J. W., Lele, S. K., and Ham, F. E., "Towards Best Practices for Jet Noise Predictions with Unstructured Large Eddy Simulations," AIAA paper 2012-2965, 2012.

${ }^{12}$ Lockard, D. P., "An efficient, two-dimensional implementation of the Ffowcs Williams and Hawkings equation," J. Sound Vib., Vol. 229, 2000, pp. 897-911. 
${ }^{13}$ Ffowcs Williams, J. E. and Hawkings, D. L., "Sound generation by turbulence and surfaces in arbitrary motion," Philosophical Transactions of the Royal Society of London. Series A, Mathematical and Physical Sciences, Vol. 264, 1969, pp. 321342 .

${ }^{14}$ Freund, J. B., "Proposed Inflow/Outflow Boundary Condition for Direct Computation of Aerodynamic Sound," AIAA J., Vol. 35, No. 4, 1997, pp. 740-742.

${ }^{15} \mathrm{Mani}$, A., "Analysis and optimization of numerical sponge layers as a nonreflective boundary treatment," Journal of Computational Physics, Vol. 231, 2012, pp. 704-7016.

${ }^{16}$ Vreman, A., "An eddy-viscosity subgrid-scale model for turbulent shear flow: Algebraic theory and applications," Physics of Fluids, Vol. 16, Jan 2004, pp. 3570.

${ }^{17}$ Kawai, S. and Larsson, J., "Wall-modeling in large eddy simulation: Length scales, grid resolution, and accuracy," Phys. Fluids, Vol. 24(015105), 2012.

${ }^{18}$ Bodart, J. and Larsson, J., "Wall-modeled large eddy simulation in complex geometries with application to high-lift devices," Annual Research Briefs, Center for Turbulence Research, Stanford University, Stanford, CA, 2011.

${ }^{19}$ Bodart, J. and Larsson, J., "Wall-modeled large eddy simulation of the McDonnell-Douglas 30P/30N high-lift airfoil in near-stall conditions," AIAA paper 2012-3022, 2012.

${ }^{20}$ Shur, M. L., Spalart, P. R., and Strelets, M. K., "Noise prediction for increasingly complex jets. Part I: Methods and tests," Int. J. Aeroacoust., Vol. 4, No. 3-4, 2005, pp. 213-246.

${ }^{21}$ Juvé, D., Sunyach, M., and Comte-Bellot, G., "Filtered azimuthal correlations in the acoustic far field of a subsonic jet," AIAA J., , No. 17, 1979, pp. 112-113.

${ }^{22}$ Kopiev, V., Chernyshev, S., Faranosov, G., Zaitsev, M., and Belayev, I., "Correlations of jet noise azimuthal components and their role in source identification," AIAA paper 2010-4018, 2010.

${ }^{23}$ Cavalieri, A. V. G., Daviller, G., Comte, P., Jordan, P., Tadmor, G., and Gervais, Y., "Using large eddy simulation to explore sound-source mechanism in jets," J. Sound Vib., Vol. 330, 2011, pp. 4098-4113.

${ }^{24}$ Cavalieri, A. V. G., Jordan, P., Colonius, T., and Gervais, Y., "Axisymmetric superdirectivity in subsonic jets," J. Fluid Mech., Vol. 704, 2012, pp. 388-420.

${ }^{25}$ Lorteau, M., Cléro, F., and F., V., "Analysis of noise radiation mechanisms in a hot subsonic jet from a validated large eddy simulation solution," Phys. Fluids, Vol. 27, 2015, pp. 075108.

${ }^{26}$ Juvé, D., Sunyach, M., and Comte-Bellot, G., "Intermittency of the noise emission in subsonic cold jets," J. Sound Vib., Vol. 71, 1980, pp. 319-332.

${ }^{27}$ Bogey, C. and Bailly, C., "Influence of nozzle-exit boundary-layer conditions on the flow and acoustic fields of initially laminar jets," J. Fluid Mech., Vol. 663, 2010, pp. 507-538.

${ }^{28}$ Bogey, C., Mardsen, O., and Bailly, C., "Influence of initial turbulence level on the flow and sound fields of a subsonic jet at a diameter-based Reynolds number of $10^{5}, "$ J. Fluid Mech., Vol. 701, 2012, pp. 352-385.

${ }^{29}$ Mendez, S., Shoeybi, M., Lele, S. K., and Moin, P., "On the use of the Ffowcs Williams-Hawkings equation to predict far-field jet noise from large-eddy simulations," Int. J. Aeroacoust., Vol. 12, 2013, pp. 1-20.

${ }^{30}$ Fosso Pouangué, A., Sanjosé, M., Moreau, S., Daviller, D., and Deniau, H., "Subsonic jet noise simulations using both structured and unstructured grids," AIAA J., Vol. 53, No. 1, 2015, pp. 55-69.

${ }^{31} \mathrm{Fu}$, Z., Agarwal, A., Cavalieri, A. V. G., Jordan, P. ., and Brès, G. A., "On removing the near-field coherent structures in a jet and its impact on the radiated sound," 22nd AIAA/CEAS Aeroacoustics Conference, Lyon, France, 2016.

${ }^{32}$ Towne, A., Colonius, T., Jordan, P., Cavalieri, A. V. G., and Brès, G. A., "Stochastic and nonlinear forcing of wavepackets in a Mach 0.9 jet," AIAA paper 2015-2217, 2015.

${ }^{33}$ Zhang, M., Tissot, G., Jordan, P., Lehnasch, G., Cavalieri, A. V. G., Colonius, T., and Brès, G. A., "Non-linear wavepacket forcing for jet noise," 21st AIAA/CEAS Aeroacoustics Conference, Aviation Forum 2015, Dallas TX, 2015.

${ }^{34}$ Cavalieri, A. V. G., Sasaki, K., Schmidt, O., Colonius, T., Jordan, P., and Brès, G. A., "High-frequency wavepackets in turbulent jet," 22nd AIAA/CEAS Aeroacoustics Conference, Lyon, France, 2016.

${ }^{35}$ Towne, A., Cavalieri, A. V. G., Jordan, P., Colonius, T., Jaunet, V., Schmidt, O., and Brès, G. A., "Trapped acoustic waves in the potential core of subsonic jets," 22nd AIAA/CEAS Aeroacoustics Conference, Lyon, France, 2016.

${ }^{36}$ Schmidt, O., Towne, A., Colonius, T., Jordan, P., Jaunet, V., Cavalieri, A. V. G., and Brès, G. A., "Super- and multidirective acoustic radiation by linear global modes of a turbulent jet," 22nd AIAA/CEAS Aeroacoustics Conference, Lyon, France, 2016.

${ }^{37}$ Jaunet, V., Jordan, P., Cavalieri, A. V. G., Towne, A., Colonius, T., Schmidt, O., and Brès, G. A., "Tonal dynamics and sound in free and installed turbulent jets," 22nd AIAA/CEAS Aeroacoustics Conference, Lyon, France, 2016. 